\title{
Efeitos de um Treino Cognitivo na Qualidade de Vida e no Bem-Estar Psicológico de Idosos
}

\author{
Effects of a Cognitive Training on the Quality of Life \\ and Well-Being of Healthy Elders
}

\author{
Tatiana Quarti Irigaray, ${ }^{*}$, , Rodolfo Herberto Schneider ${ }^{a}, \&$ Irenio Gomes $^{a}$ \\ a Pontifícia Universidade Católica do Rio Grande do Sul, Porto Alegre, Brasil
}

\begin{abstract}
Resumo
Introdução: A funcionalidade cognitiva de idosos está relacionada à sua saúde, à qualidade de vida e ao bem-estar psicológico, sendo considerada um indicador importante de envelhecimento ativo e longevidade. Objetivo: Verificar os efeitos de um treino cognitivo na qualidade de vida e no bem-estar psicológico de idosos. Métodos: 76 idosos saudáveis, divididos em grupo experimental (GE) e grupo controle (GC). O GE recebeu doze sessões de treino cognitivo, que envolvia a instrução e prática de exercícios. Resultados: Após o treino, os idosos do GE apresentaram melhor desempenho cognitivo, melhor percepção de qualidade de vida e maiores índices de bem-estar psicológico. Conclusão: Conclui-se que intervenções cognitivas que incluem tarefas de atenção, memória e funções executivas podem contribuir para a melhora da qualidade de vida e do bem-estar psicológico de idosos.

Palavras-chave: Treino Cognitivo; Qualidade de Vida; Bem-Estar Psicológico; Envelhecimento.

Abstract

Introduction: The cognitive functioning of the elderly is related to their health, quality of life and psychological well-being and is considered an important indication of active aging and longevity. Objective: To verify the effects of a cognitive training program on the quality of life and psychological well-being of the elderly. Methods: 76 healthy elderly individuals were divided into experimental group (EG) and control group (CG). The EG received 12 sessions of cognitive training that involved exercise instruction and practice. Results: After training, the elderly from EG presented better cognitive performance, better perception of quality of life and better scores of psychological well-being. Conclusion: We conclude that cognitive interventions that include attention, memory and executive function tasks can contribute to the improvement of quality of life and psychological well-being of the elderly.

Keywords: Cognitive Training; Quality of Life; Psychological Well-Being; Aging.
\end{abstract}

A boa funcionalidade cognitiva de idosos é considerada um indício importante de envelhecimento ativo e longevidade (Smits, Deeg, \& Schmand, 1999). Segundo estudos, declínios nas habilidades cognitivas de idosos levam a um aumento no risco de dificuldades no desempenho de atividades instrumentais de vida diária (Stuck et al., 1999; Yassuda \& Abreu, 2006). O bom funcionamento cognitivo é importante para a autonomia e para a capacidade de autocuidado de idosos, influenciando também nas decisões a respeito da possibilidade de o idoso continuar a viver independentemente, com segurança, dirigir seu automóvel, cuidar de suas finanças e administrar suas medicações (Yassuda \& Abreu, 2006).
Estudos mostram que o treino cognitivo pode auxiliar na manutenção e no aumento das funções cognitivas (Ball et al., 2002; Cavallini, Pagnin, \& Vecchi, 2003; O'Hara et al., 2007; Willis et al., 2006), bem como influenciar a qualidade de vida e o bem-estar psicológico de idosos, contribuindo para uma velhice bem-sucedida (Levine et al., 2007; Winocur et al., 2007; Wolinsky et al., 2006). De acordo com o Grupo de Qualidade de Vida da Organização Mundial de Saúde (The WHOQOL Group, 1998), a qualidade de vida é definida como a percepção que o indivíduo tem sobre a sua posição na vida no contexto de sua cultura e de acordo com os sistemas de valores da sociedade em que vive e em relação aos seus objetivos, as suas expectativas, aos seus padrões e às suas preocupações.

Segundo Lawton (1991), a qualidade de vida na velhice refere-se a uma avaliação multidimensional, que é realizada a partir de critérios intrapessoais e socionormativos a respeito do sistema pessoa-ambiente de um indivíduo, no momento atual, no passado e no futuro. 
Para ele, a qualidade de vida na velhice é dependente de muitos elementos que estão inter-relacionados, sendo o produto de uma história interacional que se iria delineando à medida que os indivíduos e as sociedades se desenvolvem. Ela envolveria uma comparação entre critérios objetivos e subjetivos, associados a normas e a valores sociais e individuais, os quais estariam igualmente sujeitos a alterações no decorrer do tempo. O modelo de Lawton (1983) propõe que a avaliação de qualidade de vida na velhice deve ser feita sobre quatro dimensões sobrepostas e inter-relacionadas das quais dependeria a funcionalidade do idoso: condições ambientais, competência comportamental, qualidade de vida percebida e bem-estar psicológico.

O bem-estar psicológico é definido como a capacidade do indivíduo de possuir uma visão positiva sobre si mesmo e sobre a sua vida, sensos de domínio e de autonomia, relações de qualidade com os outros, senso de propósito e de significado da vida e ter um desenvolvimento continuado na velhice. O conceito de bem-estar psicológico está relacionado ao ajustamento emocional e social, na medida em que engloba o cumprimento de tarefas evolutivas e de expectativas sociais, considerando-se seus atributos físicos, cognitivos, afetivos, idade e gênero. É a busca de crescimento pessoal, de autorealização, de constante aperfeiçoamento para manter o crescimento e o desenvolvimento. A consciência dessa busca e do constante deslocamento de metas em relação a objetivos mais elevados, favorece o ajustamento do indivíduo e a maturidade individual (Keyes, Shmotkin, \& Ryff, 2002; Ryff, 1989). Segundo Queroz e Neri (2005), a geratividade faz parte do conceito de bem-estar psicológico. A geratividade referese à motivação $\mathrm{e}$ ao envolvimento com a continuidade $\mathrm{e} o$ bem-estar do indivíduo em particular, de grupos humanos, da sociedade de modo geral e de toda a humanidade. Ela se origina da necessidade interna do indivíduo de garantir a própria imortalidade, de ser necessário e de deixar um legado para a geração seguinte (Neri, 2005).

Especificamente na velhice, ainda não há um consenso sobre a ocorrência de declínios e ganhos em relação ao bem-estar psicológico. De acordo com Ryff e Keyes (1995), com o avançar da idade, ocorrem declínios nos domínios de bem-estar psicológico: propósito na vida e crescimento pessoal e aumento nos domínios: eficácia no ambiente e autonomia, contudo a auto-aceitação parece não mudar com a idade. De forma diferente, Keyes et al. (2002) verificaram que o bem-estar psicológico aumenta com a idade, com o nível de educação e com a extroversão.

Estudos sobre a relação entre treino cognitivo, qualidade de vida e bem-estar psicológico em idosos ainda são poucos. Entre os publicados, verifica-se aumento no bem-estar psicológico após treino cognitivo, demonstrando que os benefícios do treino se estendem para outros domínios, com o bem-estar psicológico (Levine et al., 2007; Winocur et al., 2007).

De modo complementar, Wolinsky et al. (2006) sustentam que as intervenções cognitivas funcionam como fatores protetores contra os declínios na qualidade de vida na velhice. De forma diferente Winocur et al. (2007) afirmam que a qualidade de vida contribui para o envelhecimento bem-sucedido e, mais especificamente, para a preservação do funcionamento cognitivo. Deste modo, o presente estudo objetivou investigar os efeitos de um treino cognitivo na qualidade de vida e no bem-estar psicológico de idosos saudáveis.

\section{Métodos}

\section{Participantes}

A amostra constituiu-se de 76 idosos, sendo $90,8 \%$ do sexo feminino e $9,2 \%$ do sexo masculino, que foram divididos em grupo experimental (GE) e grupo controle (GC). O GE constituiu-se de 38 idosos, sendo 33 (86,8\%) do sexo feminino e $5(13,2 \%)$ do masculino, com idades entre 60 a 88 anos $(M=68,87 ; D P=7,41)$. O GC foi composto por 38 idosos, sendo $36(94,7 \%)$ do sexo feminino e $2(5,3 \%)$ do masculino, com idades entre 60 a 89 anos $(M=69,03 ; D P=6,77)$. Os dois grupos não diferiram de maneira significativa em relação ao sexo e idade dos participantes. Os indivíduos foram recrutados através da técnica de amostragem por conveniência em três grupos de convivência de idosos de Porto Alegre, em que realizavam atividades cognitivas, físicas e sociais. Eles eram idosos autônomos, socialmente ativos e residentes na comunidade. Para participar do estudo, os indivíduos deveriam ter 60 anos ou mais, participar de um dos três grupos e apresentar pontuação superior a 18 no Miniexame do Estado mental (MEEM) para idosos com baixa/ média escolaridade e $>26$ para idosos com alta escolaridade (Bertolucci, Brucki, Campaci, \& Juliano, 1994); pontuação inferior a cinco pontos na Escala de Depressão Geriátrica (GDS-15) e; pontuação inferior a 20 pontos no Inventário de Ansiedade de Beck (BAI). Foram excluídas da amostra duas idosas por apresentaram pontuação inferior ao ponto de corte sugerido no MEEM, cinco por apresentarem pontuação $>5$ pontos na GDS-15 e três por demonstrarem escore $>20$ pontos no BAI.

\section{Instrumentos}

Ficha de Dados Sociodemográficos. A ficha de dados sociodemográficos incluiu as seguintes variáveis: sexo, idade, estado civil, escolaridade, renda, percepção de saúde e número de participação em grupos de idosos.

Miniexame do Estado Mental (MEEM). O MEEM é um instrumento de rastreio cognitivo e foi empregado neste estudo com o objetivo de excluir os idosos que apresentassem critérios positivos para demência. Foi utilizada a versão em português traduzida por Bertolucci et al. (1994).

Escala de Depressão Geriátrica (GDS-15). A GDS-15 (Yesavage et al., 1983) é uma medida utilizada para identificação e para quantificação de sintomas depressivos em idosos e foi utilizada na presente pesquisa para excluir os idosos com sintomas depressivos, o que corresponde a escores $>5$ pontos. 
Inventário de Ansiedade de Beck (BAI). O BAI (Cunha, 2001) é uma medida de intensidade de ansiedade e foi aplicado no presente estudo com o intuito de excluir da amostra indivíduos com sintomas de ansiedade, ou seja, pontuação $>20$ pontos.

Instrumento de Avaliação Neuropsicológica Breve (NEUPSILIN). O NEUPSILIN é um instrumento de avaliação neuropsicológica breve, composto por 32 tarefas que avaliam 8 funções cognitivas: orientação têmporoespacial, atenção, percepção, memória, habilidades aritméticas, linguagem, praxias e funções executivas (Fonseca, Salles, \& Parente, 2009).

WHOQOL - bref. É um instrumento que avalia quatro domínios de qualidade de vida (físico, psicológico, relações sociais e meio ambiente), incluindo questões de avaliação global de qualidade de vida que geram um escore global, chamado qualidade de vida geral (Fleck et al., 2000).

Escala de Desenvolvimento Pessoal (EDEP). É um instrumento que foi construído por Neri (1999), a partir das seis subescalas de bem-estar psicológico construídas por Ryff e colaboradores (Ryff, 1989; Ryff \& Keyes, 1995), às quais foram acrescentados 12 itens que avaliam o conceito de geratividade, totalizando 30 itens. Destes, 18 refletem as escalas Ryff (três itens para cada dimensão definida pela autora), referindo-se às dimensões relações positiva com outros, autonomia, propósito de vida, crescimento pessoal, autoaceitação, domínio do ambiente, e 12 refletem o conceito de geratividade e os seus componentes criar, manter e oferecer. Cada item é avaliado por uma escala, contendo cinco pontos (1- pouquíssimo; 2pouco; 3- mais ou menos; 4- muito; 5- muitíssimo).

\section{Procedimentos}

Procedimentos para Coleta dos Dados. Primeiramente, o projeto foi examinado e aprovado pelo Comitê de Ética em Pesquisa da Pontifícia Universidade Católica do Rio Grande do Sul (PUCRS), Porto Alegre, RS, Brasil, sob o $\mathrm{n}^{\circ} .07 / 03730$. Após, foram realizados contatos com os grupos de idosos, procedendo-se, assim, à inclusão dos participantes na amostra. O presente estudo foi realizado em três etapas.

$\mathrm{Na}$ primeira etapa, foi realizada uma entrevista individual, na qual cada idoso preencheu o Termo de Consentimento Livre e Esclarecido e respondeu a informações sociodemográficas, funções cognitivas (MEEM e NEUPSILIN), sintomas depressivos (GDS-15), sintomas de ansiedade (BAI), percepção de qualidade de vida (WHOQOL-bref) e bem-estar-psicológico (EDEP). Os instrumentos foram aplicados por três psicólogas e uma aluna do curso de Psicologia da Universidade Federal do Rio Grande do Sul (UFRGS), que tinham experiência em avaliação psicológica e foram treinadas para aplicação dos instrumentos. Foram avaliados 120 idosos, sendo excluídas dez idosas por não atenderem aos critérios de inclusão do presente estudo.

$\mathrm{Na}$ segunda etapa, os 110 participantes legíveis para o estudo foram divididos de forma não-aleatória em GE e
GC, com 55 participantes em cada grupo. Optou-se por dividir os grupos de forma não-aleatória na tentativa de controlar que os dois grupos iriam possuir algum tipo de atividade cognitiva. O GE foi composto por indivíduos que participavam de dois grupos de idosos, que não realizavam nesses grupos atividades cognitivas. O GC englobou apenas participantes de um terceiro, que incluía idosos que já realizavam atividades objetivando melhora do funcionamento cognitivo global, com atividades semanais com a duração de $2 \mathrm{~h} 30 \mathrm{~min}$. Dos 55 indivíduos do GE, apenas 52 começaram as sessões de treino e desses somente 38 concluíram a intervenção. Os idosos do GE foram subdivididos em quatro grupos menores, compostos por 10 a14 idosos, a fim de facilitar a interação e o treinamento. Foram oferecidas doze sessões de intervenção para o GE, sendo quatro sessões de treino de atenção, quatro de treino de memória e quatro de treino de funções executivas. As sessões de treino foram coordenadas por uma psicóloga e realizadas através de uma sessão por semana, de 90 minutos cada. Os procedimentos foram repetidos da mesma maneira em todos os quatros grupos experimentais.

As sessões de treino de atenção foram fundamentadas no trabalho de Ostrosky-Solís e Gutiérrez (2006), que propuseram técnicas de reabilitação neuropsicológica da atenção para idosos saudáveis ou com alguma patologia. Já as sessões de treino de memória replicam, de maneira exata, o estudo de Yassuda, Batistoni, Fortes e Neri (2006), que trabalharam com treino de memória em idosos saudáveis. Já as sessões de treino de funções executivas foram inspiradas no trabalho de Sammer, Reuter, Hullmann, Kaps e Vaitl (2006), que realizaram um estudo sobre treino de funções executivas em idosos com Doença de Parkinson, uma vez que não se encontrou outros trabalhos sobre treino de funções executivas em idosos.

As sessões de treino foram divididas em três partes de aproximadamente 30 minutos cada. Na primeira parte, houve exposição teórica com abertura para discussão sobre os conceitos e tipos de atenção, memória e funções executivas e sua relação com o processo de envelhecimento. A segunda parte das sessões envolveu a execução de exercícios práticos de atenção, de memória episódica e de funções executivas. A terceira parte compreendeu a apresentação e a discussão de tarefas realizadas em casa. Os participantes realizavam leituras e exercícios em casa, após cada sessão, a fim de se prepararem para o próximo encontro. Para a leitura foram indicados capítulos do livro Deu Branco (Alvarez, 2007). Cada participante recebeu cópia do material utilizado em cada sessão, bem como cópia dos textos a ser lidos em casa. A cada sessão de treino, eles recebiam os textos e uma folha com os números das páginas a ser lidas e questões para guiar a leitura.

$\mathrm{Na}$ terceira etapa, todos os participantes (GE e GC) foram reavaliados imediatamente após o treino com os mesmos instrumentos utilizados na primeira etapa da pesquisa. No pós-teste, foram reavaliados os 38 idosos do GE que terminaram a intervenção e 40 indivíduos do GC, 

de Idosos.

do qual duas participantes foram excluídas, uma por apresentar menos de 60 anos e a outra por estar com problemas na mão direita, o que resultava em dificuldades nas tarefas que envolviam escrita. A participante com menos de 60 anos foi detectada apenas nessa etapa, em razão dos locais de recrutamento da amostra terem garantido, na etapa inicial da pesquisa, que todos os participantes tinham 60 anos ou mais. Por isso, esse dado não foi conferido na primeira etapa, apenas os escores do MEEM, GDS e BAI (critérios de exclusão). Dos 55 idosos do GC, foram reavaliados apenas 40 indivíduos, porque 15 deles não puderam comparecer à entrevista devido a problemas de saúde, viagem ou por não estarem mais participando do grupo. Assim, o número de idosos incluídos na análise foi de 76.

Procedimentos para Análise dos Dados. Os protocolos dos testes foram conferidos por duas pesquisadoras diferentes, e o grau de concordância entre as duas foi calculado, estando em torno de $99 \%$. Durante a fase de levantamento dos escores e de digitação dos dados, foi utilizado o método de avaliadores cegos, ou seja, as pesquisadoras não sabiam de quem eram os protocolos e de qual grupo os participantes pertenciam, uma vez que os protocolos foram identificados apenas através de números.
A descrição das variáveis foi realizada por meio das freqüências absolutas e relativas, bem como média e desvio-padrão. Para comparação dos valores das variáveis foi utilizado o teste $t$ para medidas repetidas e o teste $t$ para amostras independentes, uma vez que as variáveis apresentaram distribuição normal. Comparações estatísticas realizadas entre o GE e GC demonstraram que os grupos diferiam estatisticamente em relação às variáveis anos de escolaridade, renda e número de grupos de convivência de idosos dos quais participavam. Assim, utilizou-se a análise de regressão linear múltipla, sendo o valor de $P$ ajustado para anos de escolaridade, renda e número de grupos dos quais participam. Para análise dos dados, foi utilizado o programa SPSS para ambiente Windows, versão 13 .

\section{Resultados}

A Tabela 1 mostra a caracterização da amostra de acordo com os grupos de idosos. Conforme se pode verificar, os dois grupos foram formados, em sua maioria, por indivíduos casados ou viúvos. Os grupos diferiram significativamente quanto à escolaridade, à renda e ao número de grupos dos quais os idosos participam. Dessa forma, a

Tabela 1

Caracterização da Amostra quanto aos Dados Sociodemográficos, conforme os Grupos

\begin{tabular}{|c|c|c|c|}
\hline Variáveis & $\begin{array}{r}\text { GE } n(\%) \\
n(\%)\end{array}$ & $\begin{array}{r}\mathrm{GC} n(\%) \\
\quad n(\%)\end{array}$ & $p$ \\
\hline $\begin{array}{l}\text { Sexo } \\
\text { Feminino } \\
\text { Masculino }\end{array}$ & $\begin{array}{r}33(86,8) \\
5(13,2)\end{array}$ & $\begin{array}{r}36(94,7) \\
2(5,3)\end{array}$ & $0,430 * *$ \\
\hline Idade, média (desvio-padrão) & $68,87(7,41)$ & $69,03(6,77)$ & $0,923 * * *$ \\
\hline $\begin{array}{l}\text { Estado Civil } \\
\text { Solteiro(a) } \\
\text { Casado(a) } \\
\text { Separado/divorciado(a) } \\
\text { Viúvo(a) }\end{array}$ & $\begin{array}{r}2(5,3) \\
16(42,1) \\
9(23,7) \\
11(28,9)\end{array}$ & $\begin{array}{r}3(7,9) \\
12(31,6) \\
7(18,4) \\
16(42,1)\end{array}$ & $0,583^{*}$ \\
\hline $\begin{array}{l}\text { Escolaridade } \\
\quad \text { Fundamental } \\
\text { Médio } \\
\text { Superior }\end{array}$ & $\begin{array}{r}8(21,1) \\
17(44,7) \\
13(34,2)\end{array}$ & $\begin{array}{r}6(15,8) \\
9(36,8) \\
23(60,5)\end{array}$ & $0,063^{*}$ \\
\hline Anos de Escolaridade, média (desvio-padrão) & $11,47(4,22)$ & $14,16(3,87)$ & $0,005^{* * *}$ \\
\hline $\begin{array}{l}\text { Renda } \\
\qquad \begin{array}{l}1 \text { a } 4 \text { salários mínimos } \\
5 \text { a } 8 \text { salários mínimos } \\
9 \text { salários mínimos ou mais }\end{array}\end{array}$ & $\begin{array}{r}17(44,7) \\
16(42,1) \\
5(13,2)\end{array}$ & $\begin{array}{r}8(21,1) \\
14(36,8) \\
16(42,1)\end{array}$ & $0,010^{*}$ \\
\hline $\begin{array}{l}\text { Percepção de Saúde } \\
\text { Saudável } \\
\text { Doente }\end{array}$ & $\begin{array}{r}35(92,1) \\
3(7,9)\end{array}$ & $\begin{array}{r}37(97,4) \\
1(2,6)\end{array}$ & $0,615 * *$ \\
\hline $\begin{array}{l}\text { Número de grupos de convivência dos quais } \\
\text { participam, média (desvio-padrão) }\end{array}$ & $1,18(1,13)$ & $2,50(0,60)$ & $0,000 * * *$ \\
\hline
\end{tabular}

Notas. *X2 (qui-quadrado); **Fisher; *** teste $t$. 
escolaridade predominante no GE foi o ensino médio $(44,7 \%)$ e no GC o superior $(60,5 \%)$ e a renda mais presente no GE foi de 1 a 4 salários mínimos (44,7\%) e no GC foi de 9 salários mínimos ou mais $(42,1 \%)$. Quanto à participação em grupos de convivência, o GE participa em média de 1,18 grupos $(D P=1,13)$ e o $\mathrm{GC}$ de 2,50 $(D P=0,60)$. A percepção de saúde predominante em ambos os grupos foi a de saudável, sendo que o GE apresentou uma média de respostas de 92,1 e o GC de 97,4.

Através do teste $t$ para amostras pareadas, verificou-se uma diferença significativa no desempenho do GE entre o pré e pós-teste nos escores totais dos subtestes do NEUPSILIN de atenção $(p=<0,001)$, memória $(p=<0,001)$, linguagem $(p=<0,001)$, praxias $(p=<0,001)$ e funções executivas: resolução de problemas $(p=0,005)$ e fluência verbal $(p=0,015)$, mostrando melhor desempenho nessas variáveis após o treino cognitivo. De maneira geral, o GC manteve médias semelhantes em todos os subtestes do NEUPSILIN, não sendo encontrada diferença significativa entre o pré e pós-teste.
Os resultados da comparação entre os grupos no pré e pós-teste e os domínios de qualidade de vida (WHOQOLbref) são apresentados na Tabela 2. Por meio do teste $t$ para medidas repetidas, foi possível verificar diferenças estatisticamente significativas entre o pré e pós-teste no GE em relação aos domínios de qualidade de vida físico $(p<0,001)$, psicológico $(p<0,001)$ e qualidade de vida geral $(p=0,004)$. Esses resultam mostram que o GE apresentou melhor percepção de qualidade de vida nos domínios físico, psicológico e qualidade de vida geral após a intervenção. $\mathrm{O}$ teste $t$ para amostras independentes mostrou diferenças significativas entre os grupos no pré-teste nos domínios psicológico $(p=0,047)$, relacionamentos sociais $(p=0,035)$ e meio ambiente $(p=0,022)$, sendo que o GE apresentava pior percepção de qualidade de vida nesses domínios antes do treino cognitivo. A média da diferença entre os grupos no pré e pós-teste foi verificada através da análise de regressão linear múltipla, que mostrou aumento nos escores dos domínios psicológico $(p=0,010)$ e meio ambiente $(p=0,048)$ do GE com a intervenção.

Tabela 2

Comparação entre os Grupos GE e GC no Pré e Pós-Teste e os Domínios de Qualidade de Vida (WHOOL-bref)

\begin{tabular}{lcccr}
\hline Variáveis do WHOQOL-bref & $\begin{array}{c}\text { Pré-teste } \\
M \pm D P\end{array}$ & $\begin{array}{c}\text { Pós-teste } \\
M \pm D P\end{array}$ & $p^{*}$ & $\begin{array}{c}\text { Diferença } \\
M \pm D P\end{array}$ \\
\hline Domínio Físico & & & \\
$\quad$ Grupo Experimental & $71,99 \pm 17,90$ & $79,79 \pm 10,84$ & $<0,001$ & $7,80 \pm 12,34$ \\
$\quad$ Grupo Controle & $75,75 \pm 16,82$ & $75,85 \pm 15,50$ & 0,958 & $0,09 \pm 10,91$ \\
$p^{* *}$ & 0,348 & 0,202 & - & $0,122^{\#}$ \\
Domínio Psicológico & & & & \\
$\quad$ Grupo Experimental & $67,65 \pm 17,87$ & $75,11 \pm 11,92$ & $<0,001$ & $7,46 \pm 10,71$ \\
$\quad$ Grupo Controle & $74,56 \pm 11,03$ & $74,23 \pm 11,82$ & 0,812 & $-0,33 \pm 8,47$ \\
$\quad p^{* *}$ & 0,047 & 0,748 & - & $0,010^{\#}$ \\
Domínio Relacionamentos Sociais & & & & \\
$\quad$ Grupo Experimental & $69,08 \pm 21,83$ & $73,47 \pm 16,87$ & 0,113 & $4,39 \pm 16,64$ \\
$\quad$ Grupo Controle & $78,29 \pm 14,82$ & $80,26 \pm 14,29$ & 0,352 & $1,97 \pm 12,91$ \\
$\quad p^{* *}$ & 0,035 & 0,062 & - & $0,482^{\#}$ \\
Domínio Meio Ambiente & & & & \\
$\quad$ Grupo Experimental & $69,24 \pm 16,17$ & $72,12 \pm 12,84$ & 0,108 & $2,88 \pm 10,79$ \\
$\quad$ Grupo Controle & $76,89 \pm 11,99$ & $76,07 \pm 11,31$ & 0,516 & $-0,82 \pm 7,71$ \\
$\quad p^{* *}$ & 0,022 & 0,159 & - & $0,048^{\#}$ \\
Qualidade de Vida Geral & & & & $6,91 \pm 13,85$ \\
$\quad$ Grupo Experimental & $73,36 \pm 17,24$ & $80,26 \pm 12,88$ & 0,004 & $6,58 \pm 17,61$ \\
$\quad$ Grupo Controle & $74,34 \pm 21,74$ & $74,41 \pm 13,86$ & 0,924 & $0,350^{\#}$ \\
$\quad p^{* *}$ & 0,827 & 0,831 & - & \\
\hline
\end{tabular}

Notas. * teste $t$ para amostras pareadas; ** teste $t$ para amostras independentes; \# valor de $P$ ajustado para escolaridade, renda e número de grupos dos quais participam através de regressão linear múltipla.

Na Tabela 3, estão apresentados os resultados da comparação entre os grupos no pré e pós-teste e os domínios da EDEP. Através do teste $t$ para medidas repetidas, foram encontradas diferenças estatisticamente significativas entre os grupos no pré e pós-teste em relação aos domínios ambiente $(p=0,039)$, crescimento pessoal $(p=0,002)$, autoaceitação $(p=0,005)$ e na dimensão criar da subescala de geratividade da EDEP. Conforme essa comparação, os idosos do GE apresentaram melhor bemestar psicológico nesses domínios após a intervenção. No 

de Idosos.

pré-teste, os grupos GE e GC diferiram significativamente em relação a dimensão criar da subescala de geratividade $(p=0,022)$ e apresentaram uma tendência à diferença significativa no domínio relações positivas com os outros $(p=0,050)$, mostrando que o $\mathrm{GC}$ demonstrava as médias mais altas nestes domínios antes da intervenção. A média da diferença entre os grupos no pré e pós-teste foi calculada através da análise de regressão linear múltipla. Se- gundo essa comparação, o GE obteve ganhos significativos com a intervenção na dimensão criar da subescala de geratividade da $\operatorname{EDEP}(p=0,003)$. Para os domínios da EDEP ambiente $(p=0,050)$ e autoaceitação $(p=0,058)$ foi encontrada uma tendência à significância em relação à diferença entre os grupos no pré e pós-teste, em que a média de ganho do GE nesta variável foi mais alta, comparativamente ao GC.

Tabela 3

Comparação entre os Grupos GE e GC no Pré e Pós-Teste e os Domínios da Escala de Desenvolvimento Pessoal (EDEP)

\begin{tabular}{|c|c|c|c|c|}
\hline Variáveis da EDEP & $\begin{array}{l}\text { Pré-teste } \\
M \pm D P\end{array}$ & $\begin{array}{l}\text { Pós-teste } \\
M \pm D P\end{array}$ & $p^{*}$ & $\begin{array}{r}\text { Diferença } \\
M \pm D P\end{array}$ \\
\hline \multicolumn{5}{|l|}{ Autonomia } \\
\hline Grupo Experimental & $67,54 \pm 16,42$ & $71,27 \pm 14,06$ & 0,127 & $3,73 \pm 14,72$ \\
\hline Grupo Controle & $70,83 \pm 13,80$ & $68,20 \pm 12,83$ & 0,210 & $-2,63 \pm 12,72$ \\
\hline$p^{* *}$ & 0,347 & 0,323 & - & $0,350^{\#}$ \\
\hline \multicolumn{5}{|l|}{ Ambiente } \\
\hline Grupo Experimental & $76,10 \pm 13,59$ & $79,82 \pm 12,20$ & 0,039 & $3,73 \pm 10,73$ \\
\hline Grupo Controle & $78,95 \pm 13,66$ & $77,19 \pm 13,52$ & 0,273 & $-1,75 \pm 9,72$ \\
\hline$p^{* *}$ & 0,365 & 0,376 & - & $0,050^{\#}$ \\
\hline \multicolumn{5}{|l|}{ Crescimento Pessoal } \\
\hline Grupo Experimental & $76,54 \pm 15,24$ & $83,77 \pm 10,78$ & 0,002 & $7,24 \pm 13,72$ \\
\hline Grupo Controle & $78,51 \pm 14,06$ & $81,14 \pm 12,21$ & 0,148 & $2,63 \pm 10,98$ \\
\hline$p^{* *}$ & 0,559 & 0,322 & - & $0,609^{\#}$ \\
\hline \multicolumn{5}{|c|}{ Relações Positivas com os Outros } \\
\hline Grupo Experimental & $69,96 \pm 19,13$ & $73,90 \pm 16,11$ & 0,062 & $3,95 \pm 12,67$ \\
\hline Grupo Controle & $77,41 \pm 12,98$ & $75,66 \pm 11,52$ & 0,384 & $-1,75 \pm 12,28$ \\
\hline$p^{* *}$ & 0,050 & 0,587 & - & $0,162^{\#}$ \\
\hline \multicolumn{5}{|l|}{ Autoaceitação } \\
\hline Grupo Experimental & $68,86 \pm 18,75$ & $74,56 \pm 15,37$ & 0,005 & $5,70 \pm 11,64$ \\
\hline Grupo Controle & $74,12 \pm 15,47$ & $76,75 \pm 13,86$ & 0,166 & $2,63 \pm 11,48$ \\
\hline$p^{* *}$ & 0,186 & 0,516 & - & $0,058^{\#}$ \\
\hline \multicolumn{5}{|l|}{ Propósito na Vida } \\
\hline Grupo Experimental & $72,81 \pm 18,55$ & $75,88 \pm 15,83$ & 0,090 & $3,07 \pm 10,86$ \\
\hline Grupo Controle & $75,88 \pm 15,11$ & $76,32 \pm 12,18$ & 0,834 & $0,44 \pm 12,84$ \\
\hline$p^{* *}$ & 0,431 & 0,893 & - & $0,545^{\#}$ \\
\hline \multicolumn{5}{|l|}{ Criar } \\
\hline Grupo Experimental & $55,92 \pm 17,96$ & $64,04 \pm 14,64$ & 0,002 & $8,11 \pm 14,94$ \\
\hline Grupo Controle & $64,69 \pm 14,43$ & $61,62 \pm 18,54$ & 0,221 & $-3,07 \pm 15,18$ \\
\hline$p^{* *}$ & 0,022 & 0,531 & - & $0,003^{\#}$ \\
\hline \multicolumn{5}{|l|}{ Oferecer } \\
\hline Grupo Experimental & $69,52 \pm 14,85$ & $68,64 \pm 13,34$ & 0,582 & $-0,88 \pm 9,74$ \\
\hline Grupo Controle & $70,28 \pm 14,08$ & $69,96 \pm 13,18$ & 0,839 & $-0,33 \pm 9,90$ \\
\hline$p^{* *}$ & 0,818 & 0,667 & - & $0,866^{\#}$ \\
\hline \multicolumn{5}{|l|}{ Manter } \\
\hline Grupo Experimental & $73,25 \pm 15,76$ & $73,68 \pm 14,31$ & 0,762 & $0,44 \pm 8,87$ \\
\hline Grupo Controle & $67,98 \pm 14,69$ & $67,54 \pm 13,81$ & 0,849 & $-0,44 \pm 14,10$ \\
\hline$p^{* *}$ & 0,136 & 0,061 & - & $0,893^{\#}$ \\
\hline
\end{tabular}

Notas. * teste $t$ para amostras pareadas; ** teste $t$ para amostras independentes; \# valor de $P$ ajustado para escolaridade, renda e número de grupos dos quais participam através de regressão linear múltipla. 


\section{Discussão}

No presente estudo, verificou-se que, após o treino cognitivo, os idosos do GE apresentaram melhor desempenho cognitivo nas funções de atenção, memória, linguagem, praxias, resolução de problemas e funções executivas, em relação aos idosos do GC. Estes dados sugerem efeitos positivos do treino nas funções cognitivas de idosos e estão de acordo com a literatura (Levine et al., 2007; Valentijn et al., 2005; Willis et al., 2006; Yassuda et al., 2006). Além disso, constatou-se que os efeitos do treino cognitivo se estenderam para outras dimensões, levando a uma melhora significativa da percepção de qualidade de vida e de bem-estar psicológico dos participantes do GE após o treino cognitivo.

No pós-teste, os idosos do GE apresentaram aumento no seu desempenho cognitivo e melhor percepção de qualidade de vida nos domínios físico, psicológico e qualidade de vida geral, comparativamente ao GC. Uma possível explicação para esse achado seria a de que existe uma associação entre bom funcionamento cognitivo em idosos e boa percepção de qualidade de vida, assim, o aumento no funcionamento cognitivo dos idosos do GE resultou em uma melhor percepção de sua qualidade de vida ou vice-versa. Estudos mostram uma associação positiva entre função cognitiva e qualidade de vida, assim, quanto mais declínio cognitivo, pior a qualidade de vida dos indivíduos (Missotten et al., 2008; Ready \& Ott, 2003). Para Ribeiro e Yassuda (2007), a capacidade cognitiva é um dos determinantes da qualidade de vida, pois as perdas cognitivas podem resultar em comprometimento do funcionamento físico, social e emocional dos idosos. Da mesma forma, Winocur et al. (2007) defende a hipótese de que a qualidade de vida contribui para o envelhecimento bem-sucedido e, mais especificamente, para preservar o funcionamento cognitivo.

Uma outra hipótese de explicação para a melhora na percepção de qualidade no GE, no pós-teste, seria a de que os participantes constituíram novas redes sociais a partir do contato com outros idosos nas sessões de treino cognitivo. Segundo Chachamovich, Trentini e Fleck (2007), o tamanho, a configuração e as funções das redes sociais são elementos associados à qualidade de vida na velhice. Já as redes sociais insatisfatórias estariam relacionadas à pior percepção subjetiva de qualidade de saúde, a piores componentes mentais de qualidade de vida relacionada à saúde em mulheres e a piores escores em componentes físicos de qualidade de vida relacionada à saúde em homens. A rede social teria a capacidade de amortecer impactos emocionais negativos, pela percepção do indivíduo de que não está sozinho, de que tem com quem contar, sentindo segurança decorrente do senso de pertencimento e de integração (Garcia, Banegas, \& Pérez-Regadera, 2005). Para Argimon, Stein, Xavier e Trentini (2004), a criação de redes sociais provedoras de apoio ao idoso favorecem as suas relações sociais, bem como oferece-lhe a oportunidade de permanecer ativo e de estabelecer novos contatos sociais.

Observou também um aumento no bem-estar psicológico dos idosos do GE, no pós-teste, nos domínios ambiente, crescimento pessoal, autoaceitação e na dimensão criar da subescala de geratividade. Freire (2000) define o crescimento pessoal como ter um senso de crescimento contínuo e de desenvolvimento como pessoa, estar aberto a novas experiências, ter um senso de realização do potencial, cujas mudanças refletem em autoconhecimento e eficácia. Já a autoaceitação caracteriza-se como uma atitude positiva do indivíduo em relação a si mesmo e ao seu passado e o reconhecimento e a aceitação de vários aspectos de si mesmo, tanto bons quanto ruins (Freire, 2000). Queroz e Neri (2005) definem a dimensão criar como a geração ou a criação de indivíduos, coisas, ações e idéias que sirvam para perpetuar a espécie humana, tanto no sentido biológico quanto sociocultural. A geratividade refere-se à motivação e ao envolvimento com a continuidade e o bem-estar de indivíduos particulares, de grupos humanos, da sociedade de modo geral e de toda a humanidade. Ela origina-se da necessidade interna do individuo de garantir a própria imortalidade, de ser necessário e de deixar um legado para a geração seguinte (Queroz \& Neri, 2005).

Uma associação entre treino cognitivo e melhor bemestar psicológico em idosos tem sido encontrada em outros estudos (Stuss et al., 2007; Winocur et al., 2007). Uma possibilidade de explicação para essa associação encontrada no presente estudo seria a de que o funcionamento cognitivo de idosos é influenciado pelo bem-estar psicológico ou vice-versa. Por isso, os benefícios do treino cognitivo se estenderam para domínios de bem-estar psicológico, evidenciando uma importante relação entre fatores psicossociais e desempenho cognitivo de idosos. Para Winocur et al. (2007), os fatores psicossociais relacionam-se com o funcionamento cognitivo de idosos, evidenciando que tanto fatores biológicos quanto nãobiológicos contribuem para a função cognitiva e conquista de um envelhecimento bem-sucedido.

Segundo Hultsch, Hertzog, Small e Dixon (1999), o engajamento em atividades intelectuais serve como um fator protetor contra o declínio cognitivo na velhice. Os mesmos autores oferecem uma hipótese alternativa, sugerindo que indivíduos com desempenho cognitivo excelente levam uma vida ativa até o declínio cognitivo começar a limitar suas atividades intelectuais na velhice. Argimon et al. (2004) defendem a hipótese de que, quanto maior a participação de idosos em atividades de lazer, melhor será seu desempenho cognitivo. O lazer e a manutenção da capacidade de realizar atividades de aptidão física e mental favorecem uma melhor qualidade de vida. Anos de escolaridade e mais opções de lazer atuam como fatores de proteção de declínio nas habilidades cognitivas de idosos.

Uma possibilidade de explicação para a melhora na percepção de qualidade de vida e no bem-estar psicoló- 
gico do GE, após a intervenção, seria a de que a participação nas sessões de treino (fator externo), desencadeou mecanismos internos psicológicos que produziram melhora no bem-estar psicológico e na qualidade de vida. De acordo com Rogerson (1995) a qualidade de vida é influenciada por dois grupos de variáveis. O primeiro corresponde aos mecanismos internos psicológicos e fisiológicos, que produzem grau de satisfação e gratificação na vida. O segundo refere-se a fatores externos, capazes de desencadear os primeiros. O conceito de bemestar psicológico está diretamente implicado na determinação de uma boa qualidade de vida em idosos, uma vez que apresenta correlação com uma série de características na velhice (Chachamovich et al., 2007). Dessa forma, o bem-estar psicológico parece ser um construto chave para o entendimento da qualidade de vida na velhice (Keyes et al., 2002).

De acordo com Freire (2000), os idosos têm importantes reservas para o desenvolvimento, que podem ser ativadas pela aprendizagem, por exercícios ou por treinamento. Em boas condições de saúde e ambientais, a maioria dos idosos continua a ter potencial para desempenho em altos níveis e para adquirir conhecimentos teóricos e práticos. Assim, envelhecer não se constitui como um fator de risco, pois os idosos se tornam mais eficazes no uso de processos adaptativos, tendem a apresentar boa compreensão sobre as condições que definem o que é possível, ou não, e a aplicar o conhecimento em seu desenvolvimento pessoal e em sua adaptação.

A partir do presente estudo, pode-se concluir que um estilo de vida cognitivamente intenso, com demandas exigentes e interessantes, leitura e prática de exercícios, parece ser um preditor para a boa qualidade de vida e para o bem-estar psicológico em idosos saudáveis. $\mathrm{O}$ treino cognitivo pode melhorar o funcionamento cognitivo de idosos e colaborar para a manutenção da boa qualidade e do bem-estar psicológico na velhice. Os resultados encontrados indicam que idosos saudáveis podem-se beneficiar desse tipo de intervenção, reduzindo a ocorrência de condições que levem a um envelhecimento cognitivo patológico e fortalecendo a manutenção da boa qualidade de vida e do bem-estar psicológico na velhice.

É importante destacar que este estudo foi conduzido com uma amostra relativamente homogênea, com nível de escolaridade e renda elevados, o que provavelmente não é a realidade de outros grupos de idosos. Possivelmente, os idosos que participam desses programas têm características distintas daqueles que não participam, como cognição e saúde mais preservadas, que favorecem o engajamento nas atividades. Assim, é necessária a realização de novos estudos a fim de explorar a associação entre participação em um treino cognitivo, qualidade de vida e bem-estar psicológico em idosos saudáveis. Além disso, há escassez na literatura sobre os efeitos psicossociais resultantes da participação de idosos em treino cognitivo.

\section{Referências}

Alvarez, A. M. M. (2007). Deu branco: Um guia para desenvolver o potencial de sua memória. Rio de Janeiro, RJ: Record.

Argimon, I. I. L., Stein, L. M., Xavier, F. M. F., \& Trentini, C. M. (2004). O impacto de atividades de lazer no desenvolvimento cognitivo de idosos. Revista Brasileira de Ciências do Envelhecimento Humano, 1(1), 38-47.

Ball, K., Berch, D. B., Helmers, K. F., Jobe, J. B., Leveck, M. D., Marsiske, M., et al. (2002). Effects of cognitive trainig interventions with olders adults: A randomized controlled trial. Journal of the American Medical Association, 288(18), 2271-2281.

Bertolucci, P. H. F., Brucki, S. M. D., Campacci, S. R., \& Juliano, Y. (1994). O mini-exame do estado mental em uma população geral: Impacto da escolaridade. Arquivos de NeuroPsiquiatria, 52(1), 1-7.

Cavallini, E., Pagnin, A., \& Vecchi, T. (2003). Aging and everyday memory: The beneficial effect of memory training. Archives of Gerontology and Geriatrics, 37(3), 241-257.

Chachamovich, E., Trentini, C., \& Fleck, M. P. A. (2007). Qualidade de vida em idosos. In A. L. Neri (Ed.), Qualidade de vida na velhice: Enfoque multidisciplinar (pp. 61-81). Campinas, SP: Alínea.

Cunha, J. A. (2001). Manual da versão em português das Escalas Beck. São Paulo, SP: Casa do Psicólogo.

Fleck, M. P. A., Louzada, S., Xavier, M., Chachamovich, E., Vieira, G., Santos, L., et al. (2000). Aplicação da versão em português do instrumento abreviado de avaliação de qualidade de vida WHOQOL-BREF. Revista de Saúde Pública, 34(2), 178-183.

Fonseca, R. P., Salles, J. F., \& Parente, M. A. M. P. (2009). Instrumento de avaliação neuropsicológica breve NEUPSILIN. São Paulo, SP: Vetor.

Freire, S. A. (2000). Envelhecimento bem-sucedido e bemestar psicológico. In A. L. Neri \& S. A. Freire (Eds.), E por falar em boa velhice (pp. 21-31). São Paulo, SP: Papirus.

Garcia, E. L., Banegas, J. R., \& Pérez-Regadera, A. G. (2005). Social network and health-related quality of life in older adults: A population-based study in Spain. Quality of Life Research, 14(2), 511-520.

Grupo de Qualidade de Vida da Organização Mundial de Saúde. (1998). The World Health Organization quality of life assessment (WHOQOL): Development and general psychometric properties. Social, Science and Medicine, 46, 1569-1585.

Hultsch, D. F., Hertzog, C., Small, B. J., \& Dixon, R. A. (1999). Use it or lose it: Engaged lifestyle as a buffer of cognitive decline in aging? Psychology and Aging, 14(2), 245-263.

Keyes, C. L. M., Shmotkin, D., \& Ryff, C. D. (2002). Optimizing well-being: The empirical encounter of two traditions. Journal of the Personality and Social Psychology, 82(6), 1007-1022.

Lawton, M. P. (1983). Environment and other determinants of well-being in older people. The Gerontologist, 23(4), 349355.

Lawton, M. P. (1991). A multidimensional view of quality of life in frail elderly. In J. E. Birren, J. E. Lubben, J. C. Rowe, \& D. E. Deutchmann (Eds.), The concept and measurement of quality of life in the frail elderly (pp. 3-27). San Diego: Academic Press. 
Levine, B., Stuss, D., Winocur, G., Binns, M. A., Fahy, L., Mandic, M., et al. (2007). Cognitive rehabilitation in the elderly: Effects on strategic behavior in relation to goal management. Journal of the International Neuropsychological Society, 13(1), 143-152.

Missotten, P., Squelard, G., Ylieff, M., Di Notte, D., Paquay, L., De Lepeleire, J., et al. (2008). Relationship between quality of life and cognitive decline in dementia. Dementia and Geriatric Cognitive Disorders, 25(6), 564-572.

Neri, A. L. (1999). Fundamentos para uma escala de geratividade. Campinas, SP: Universidade Estadual de Campinas. Relatório de pesquisa não-publicado.

Neri, A. L. (2005). Palavras-chave em gerontologia. Campinas, SP: Alínea.

O’Hara, R., Brooks, J. O., Friedman, L., Schroder, C. M., Morgan, K. S., \& Kraemer, H. C. (2007). Long-term effects of mnemonic training in community-dwelling older adults. Journal of Psychiatric Research, 41(7), 585-590.

Ostrosky-Solís, F., \& Gutiérrez, A. L. (2006). Reabilitação neuropsicológica da atenção e da memória. In J. AbrisquetaGomes \& F. H. Santos (Eds.), Reabilitação neuropsicológica: Da teoria à prática (pp. 227-240). São Paulo, SP: Artes Médicas.

Queroz, N. C., \& Neri, A. L. (2005). Bem-estar psicológico e inteligência emocional entre homens e mulheres na meiaidade e na velhice. Psicologia Reflexão e Critíca, 18(2), 292299.

Ready, R. E., \& Ott, B. R. (2003). Quality of life measures for dementia. Health and Quality of Life Outcomes, 1, 11-19.

Ribeiro, P. C. C., \& Yassuda, M. S. (2007). Cognição, estilo de vida e qualidade de vida na velhice. In A. L. Neri (Ed.), Qualidade de vida na velhice: Enfoque multidisciplinar (pp. 189204). Campinas, SP: Alínea.

Rogerson, R. J. (1995). Enviromental and health-related quality of life: Conceptual and methodological similarities. Social Science \& Medicine, 41(10), 1373-1382.

Ryff, C. D. (1989). Happiness is everything, or is it? Explorations on the meaning of psychological well-being. Journal of the Personality and Social Psychology, 57(6), 1069-1081.

Ryff, C. D., \& Keyes, C. L. M. (1995). The structure of psychological well-being revisited. Journal of the Personality and Social Psychology, 69(4), 719-727.

Sammer, G., Reuter, I., Hullmann, K., Kaps, M., \& Vaitl, D. (2006). Training of executive functions in Parkinson's disease. Journal of the Neurological Sciences, 248(1-2), 115-119.

Smits, C. H., Deeg, D. M., \& Schmand. B. (1999). Cognitive functioning and heath as determinants of mortality in an older population. American Journal of Epidemiology, 150(9), 978-986.

Stuck, A. E., Walther, J. M., Nikolaus, T., Bula, C. J., Hohmann, C., \& Beck, J. C. (1999). Risk factors for functional status decline in community-living elderly people: A systematic literature review. Social Science \& Medicine, 48(4), 445-469.

Stuss, D. T., Robertson, I. H., Craik, F. I. M., Levine, B., Alexander, M. P., Black, S., et al. (2007). Cognitive rehabilitation in the elderly: A randomized trial to evaluate a new protocol. Journal of the International Neuropsychological Society, 13(1), 120-131.

Valentijn, S. A. M., Van Hooren, S. A. H., Bosma, H., Touw, D. M., Jolles, J., Van Boxtel, M. P. J., et al. (2005). The effect of two types of memory training on subjective and objective memory performance in healthy individuals aged 55 years and older: A randomized controlled trial. Patiente Education and Counseling, 57(1), 106-114.
Willis, S. L., Tennstedt, S. L., Marsiske, M., Ball, K., Elias, J., Koepke, K. M., et al. (2006). Long-term effects of cognitive training on everyday functional outcomes in older adults. Journal of the American Medical Association, 296(23), $2805-$ 2814.

Winocur, G., Palmer, H., Dawson, D., Binns, M., Bridges, K., \& Stuss, D. (2007). Cognitive rehabilitation in the elderly: An evaluation of psychosocial factors. Journal of the International Neuropsychological Society, 13, 153-165.

Wolinsky, F., Unverzagt, F. W., Smith, D. M., Jones, R., Stoddard, A., \& Tennstedt, S. L. (2006). The ACTIVE cognitive training trial and health-related quality of life: Protection that lasts for 5 years. Journals of Gerontology Series A: Biological Science, 61A(12), 1324-1329.

Yassuda, M. S., \& Abreu V. P. S. (2006). Avaliação cognitiva. In E. V. Freitas, L. Py, F. A. X. Cançado, J. Doll, \& M. L. Gorzoni (Eds), Tratado de Geriatria e Gerontologia (pp. 1252-1266). Rio de Janeiro, RJ: Koogan.

Yassuda, M. S., Batistoni, S. S. T., Fortes, A. G., \& Neri, A. L. (2006). Treino de memória no idoso saudável: Benefícios e mecanismos. Psicologia Reflexão e Critíca, 19(3), 470-481.

Yesavage, J. A., Brink, T. L., Rose, T. L., Lum, O., Huang, V., Adey, M., et al. (1983). Development and validation of a geriatric depression screening scale: A preliminary report. Journal of Psychiatric Research, 17(1), 37-49.
Recebido: $30 / 10 / 2009$

$1^{a}$ revisão: $04 / 08 / 2010$

$2^{a}$ revisão: $20 / 08 / 2010$ Aceite final: $10 / 09 / 2010$ 